ISSN: 2637-773X

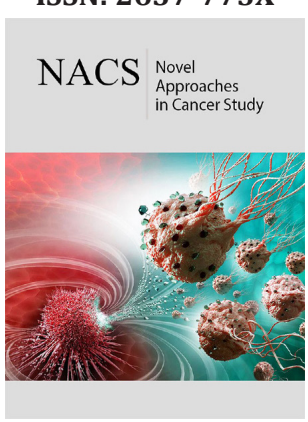

*Corresponding author: Doaa Raafat Ali, Air Force Specialized Hospital, Cairo, Egypt

Submission: 侮 May 04, 2021

Published: 監June 18, 2021

Volume 6 - Issue 1

How to cite this article: Doaa Raafat Ali, Amin El-Sayed Amin, Abdelsattar Mohamed Sallam El-Sayed Mahmoud, El-Sayed. Comparison Between VMAT and IMRT in Remaining Volume at Risk for Large Volume Head and Neck Cancer Patients. Nov Appro in Can Study. 6(1). NACS.000626. 2021. DOI: 10.31031/NACS.2021.06.000626

Copyright@ Doaa Raafat Ali, This article is distributed under the terms of the Creative Commons Attribution 4.0 International License, which permits unrestricted use and redistribution provided that the original author and source are credited.

\section{Comparison Between VMAT and IMRT in Remaining Volume at Risk for Large Volume Head and Neck Cancer Patients}

Doaa Raafat Ali ${ }^{1 *}$, Amin El-Sayed Amin², Abdelsattar Mohamed Sallam ${ }^{3}$ and ElSayed Mahmoud El-Sayed ${ }^{3}$

${ }^{1}$ Air Force Specialized Hospital, Egypt

${ }^{2}$ Oncology department, Faculty of Medicine, Ain Shams University, Cairo, Egypt

${ }^{3}$ Physics department, Faculty of Science, Ain Shams University, Cairo, Egypt

\begin{abstract}
Background: Radiation therapy for advanced head and neck cancer has developed from the ThreeDimensional Conformal Radiotherapy 3DCRT to intensity modulated radiation therapy IMRT and Volumetric Modulated Arc Therapy VMAT. Remaining Volume at Risk RVR is clinically important in plan evaluating process. RVR is useful for assisting and estimating the risk of late effects.
\end{abstract}

Aim: The aim of the present work is to compare dosimetric evaluation factors and dosimetric data remaining volume at risk for both IMRT and VMAT in the treatment of large volume head and neck cancer patients.

Patients and methods: Eleven retrospective patients with advanced head and neck tumors were selected for the planning study. The patients' treatments are planned by using the two different techniques (IMRT and VMAT) and optimized to evaluate highly conformal target coverage and sparing organs at risk OAR. Standard fractionation is 33 fractions with 5 fractions weekly, the prescribed doses 70Gy, 59.4Gy and 54Gy for the high, intermediate, and low risk targets by simultaneous integrated boost.

Results: The study showed that VMAT and IMRT are comparable in target volumes homogeneity index $\mathrm{HI}$, while VMAT is more superior $(0.76,44.4$ and 589$)$ and $(0.68,55.6$ and 1989$)$ in conformity number $\mathrm{CN}$, gradient index GI and Monitor Units (MU), respectively. Furthermore, VMAT has an advantage over IMRT in RVR volumes that received 15Gy, 10Gy and 5Gy, which are (4327, 5281 and 6703cc) and a 1019cGy mean dose in the VMAT technique, while in IMRT are (4435, 5311 and 6543cc) and a 1051cGy mean dose.

Conclusion: The results of comparing dosimetric evaluation factors of both techniques showed that VMAT had a priority over IMRT in 3 factors of RVR evaluation parameters. Additionally, to other comparison points, especially the time on the machine, makes VMAT a first choice in the treatment plan selection.

Keywords: Head and Neck; IMRT; VMAT; RVR

Abbreviation: 3DCRT: Three-Dimensional Conformal Radiotherapy; IMRT: Intensity Modulated Radiation Therapy; VMAT: Volumetric Modulated Arc Therapy; RVR: Remaining Volume at Risk; HI: Homogeneity Index; CN: Conformity Number; GI: Gradient Index; MU: Monitor Units; HNC: Head and Neck Cancer; OAR: Organs at Risk; GTV: Gross Target Volume; CTV: Clinical Target Volume; PTV: Planning Target Volume

\section{Introduction}

Head and neck cancer contains a large category of similar cancers that begin in larynx, lip, salivary glands, oral cavity, nasopharynx, pharynx, and thyroid gland (Figure 1). The most used methods for head and neck cancer treatment are surgery, chemotherapy, and radiotherapy. The radiation therapy aims to deliver a prescribed dose to target volumes, to kill the tumour cells, with a good protection of Organs at Risk OAR [1]. Tobacco and alcohol abuse and human papillomavirus infection are two primary causes of Head and Neck Cancer (HNC). The HNC commonly spreads throughout some countries around the world. The estimated percentage of $\mathrm{HNC}$ is $4 \%$ of all malignancy's tumour [2]. The difficulty of treatment planning for advanced HNC is the complex shape of target volumes with sparing critical organs like 
the brainstem, parotid glands, spinal cord, mandible, and normal structures. Due to this potential complication between the tumor and critical structures, head and neck planning presents a challenge for radiotherapy [3]. Advances in computer and linear accelerator technology has also significantly improving the treatment of HNC to maximize radiation dose to tumor while minimizing the radiation dose to surrounding normal critical structures. The development of inverse planning systems for delivering the non-uniform radiation intensities are widely used in the treatment of many HNC [4].

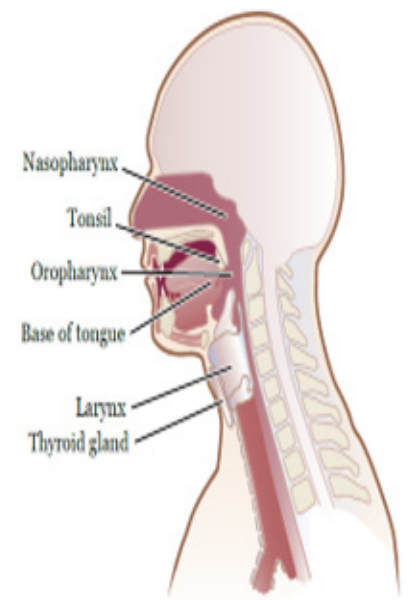

Figure 1: Head and neck cancer.

For the past years inverse planning as Intensity Modulated Radiotherapy IMRT has become available to treatment many tumors. IMRT allows for the dose of radiation to conform precisely to the tumor three-dimensional shape by controlling the intensity of the radiation beam in multiple small volumes. Treatment planning techniques for HNC have advanced from the conventional threefield technique to IMRT over two decades. Volumetric modulated arc therapy VMAT is a modern rotational IMRT that became available to compete with IMRT for HNC treatment [5]. On the other hand, IMRT and VMAT dose distribution is inversely planned, which means that the dosimetrist selects the dose distribution required for the planning target volumes and the acceptable tolerance dose for normal tissue organs [6]. In 2018, about 129,000 nasopharyngeal carcinoma patients were diagnosed and about 73,000 died. The advances of radiation technology, IMRT and VMAT have become standard radiotherapy methods for nasopharyngeal carcinoma patients [7].

The RVR is defined as the whole external contour of the patient's image minus the target volumes and OAR (Figure 2), which is recommended by the International Commission on Radiation Units 83 report [7]. The RVR is clinically important in plan evaluating process. If not specifically evaluated, there could be unsuspected areas of high absorbed dose within the patient that would go undetected. RVR may be useful for measuring low dose radiation exposure to assist and estimating the risk of late effects, such as carcinogenesis which is important for the younger patients. Global Harmonization Group consensus guidelines contoured
RVR as one of excluded structures [8]. One of the studies that compare the different techniques in prostate cancer found that the Rotational technique, like VMAT and helical tomotherapy, have an effect on the high low-dose radiation exposure to all normal tissues in the radiation field [9]. In another study concerned on the breast irradiation treatment, IMRT had the highest low dose radiation followed by the VMAT technique [10]. The aim of present work is to compare the treatment planning for the large volume of HNC patients using IMRT and VMAT techniques to evaluate low dose effect for remaining volume at risk.

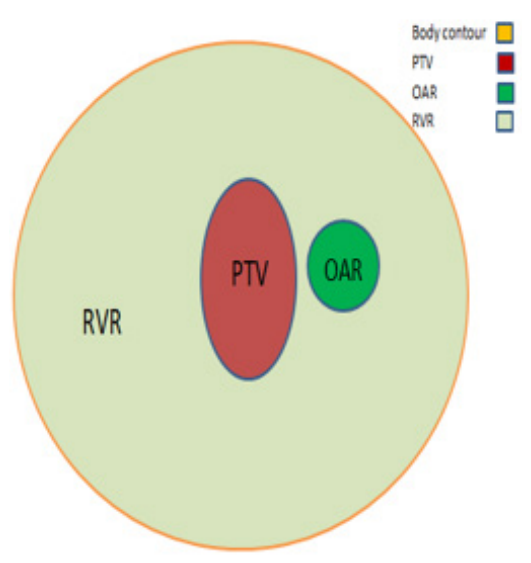

Figure 2: Remaining volume at risk.

\section{Materials and Methods}

Eleven patients with advanced HNC with previous treatment were selected for the planning study. Patients are planned to use the two different techniques and optimized to evaluate highly conformal target coverage and sparing OAR. The RVR that generated are contoured to evaluate the low dose effect that by calculating the volumes that received the low doses (V 15Gy, V 10Gy and V 5Gy). Head and neck cases contain three different target volumes (high, intermediate, and low target volume). Dose escalation between target volumes can be prepared in single plan, which uses the simultaneous integrated boosts facility to minimize planning and quality assurance time.

Standard fractionation is 33 fractions with 5 fractions weekly. The gross target volume GTV 70 is defined as all known gross disease determined by physical examination, CT, MRI, and PET. Clinical target volume (CTV, 59.4) contains an additional margin around the gross tumor, including potential sites of spread and high-risk nodal levels. CTV 54 comprises the lower risk subclinical regions (1.64Gy/fraction). The planning target volume PTV is added as a margin for each CTV [11]. Patients were immobilized in the supine position with a fully extended neck with a thermoplastic mask over the head and shoulders. CT scan of the head and neck will be taken with $3 \mathrm{~mm}$ axial cuts down to the chest region. CT is then transferred to the Eclipse treatment planning system using the Anisotropic Analytical Algorithm (AAA) for plan photon dose calculation. Plans were designed for treatment by using the True Beam linear accelerator system. 
A large volume of HNC is found in ones with radiotherapy difficulties; and therefore, using the IMRT 9 sliding window coplanar fields equally spaced with $6 \mathrm{MV}$ photons energy to reach the optimal result for plan evaluation, Figure 3(A). Optimal fluence maps from optimization process are then converted by a leaf motion calculator into actual fluence maps which are deliverable using a multi-leaf collimator. For VMAT plans, two full coplanar arcs of 6MV energy photons were used, Figure 3(B). One arc starts from a gantry angle $181^{\circ}$ and ends at gantry of $179^{\circ}$ in a clockwise direction. The other arc is rotating counterclockwise with different collimator angles. The study analysis data represent target volumes homogeneity, conformity and gradient which good indicate for the quality of plan coverage. The homogeneity index HI was evaluated as the ratio between the dose covering $95 \%$ and $5 \%$ as expressed as (D95\%/D5\%). Conformity Number CN defined as a ratio between PTV coverage at the Prescription Dose and Prescription Isodose Surface Volume PIV multiply by ratio between PTV coverage at the Prescription Dose and the Target Volume [(PTVPD/ PIV) $\mathrm{x}$ PTVPD/ PTV)]. Gradient index GI was evaluated as a ratio between prescription dose of fifty percent volume (Body V 50\%) and a Prescription Isodose Surface Volume PIV (Body V 50\% / PIV) [12].

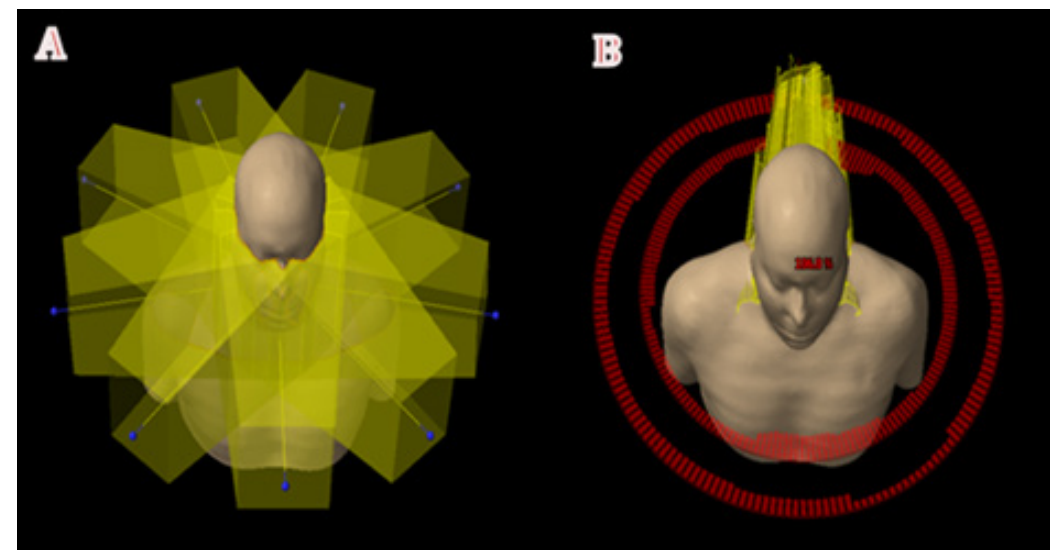

Figure 3: Treatment planning for IMRT (A) and VMAT (B).

\section{Results}

Table 1: Target volumes HI for all cases.

\begin{tabular}{|c|c|c|c|c|c|c|c|c|c|}
\hline & \multicolumn{3}{|c|}{ PTV $70 \mathrm{HI}$} & \multicolumn{3}{|c|}{ PTV 59.4 HI } & \multicolumn{3}{|c|}{ PTV 54HI } \\
\hline Case & VMAT & IMRT & VP & VMAT & IMRT & VP & VMAT & IMRT & VP \\
\hline 1 & 0.95 & 0.96 & 0.91 & 0.87 & 0.89 & 1.70 & 0.93 & 0.94 & 1.83 \\
\hline 2 & 0.95 & 0.95 & 0.37 & 0.87 & 0.88 & 0.99 & 0.95 & 0.96 & 1.12 \\
\hline 3 & 0.94 & 0.95 & 1.51 & 0.85 & 0.86 & 0.28 & 0.94 & 0.95 & 1.36 \\
\hline 4 & 0.92 & 0.96 & 4.19 & 0.84 & 0.87 & 2.76 & 0.93 & 0.95 & 1.53 \\
\hline 5 & 0.96 & 0.96 & 0.64 & 0.96 & 0.96 & 0.88 & 0.88 & 0.89 & 0.89 \\
\hline 6 & 0.96 & 0.96 & 0.91 & 0.89 & 0.91 & 2.38 & 0.95 & 0.96 & 1.72 \\
\hline 7 & 0.97 & 0.97 & 0.01 & 0.92 & 0.93 & 1.38 & 0.95 & 0.95 & -0.04 \\
\hline 8 & 0.91 & 0.95 & 4.75 & 0.84 & 0.87 & 2.92 & & & \\
\hline 9 & 0.96 & 0.97 & 1.02 & 0.87 & 0.88 & 0.39 & & & \\
\hline 10 & 0.94 & 0.96 & 2.28 & 0.89 & 0.92 & 2.94 & & & \\
\hline 11 & 0.96 & 0.96 & 0.22 & 0.87 & 0.87 & 0.05 & 0.94 & 0.95 & 1.03 \\
\hline AVG & 0.95 & 0.96 & -1.53 & 0.88 & 0.89 & -1.52 & 0.93 & 0.95 & -1.18 \\
\hline
\end{tabular}

Table Abbreviations: HI: Homogeneity Index; VMAT: Volumetric Modulated Arc Therapy; IMRT: Intensity Modulated Radiation Therapy; VP: Variance Percentage; AVG: Average. 
The study analysis of the dosimetric data showed that the HI average results of the PTV70 HI, PTV60 HI and PTV54 HI are $(0.93,0.88$ and 0.93$)$ and $(0.95,0.89$ and 0.95$)$ for VMAT and IMRT, respectively (Table 1; Figure 4). Furthermore, the CN, GI and MU study are showed $(0.76,44.4$ and 589) and $(0.68,55.6$ and 1989) for VMAT and IMRT, respectively (Table 2; Figure 5). In the same way to evaluate the low dose effect of Remaining Volume at Risk RVR, the Resultant Data of the Average Volume of RVR that received 15Gy, 10Gy and 5Gy are (4327, 5281 and 6703cc) and 1019cGy mean dose using the VMAT technique, which using the IMRT technique produced (4435, 5311 and 6543cc) and 1051cGy mean dose (Table 2; Figure 6). Figure 7 shows the variance percentage between the two techniques (HI, CN, GI, and MU). The Upper column means that VMAT has the advantage while lower columns are inversely related. On the other hand, Figure 8, show the variance percentage between the two techniques in RVR for the volume of 15Gy, 10Gy, 5Gy and RVR mean dose (Table 3).

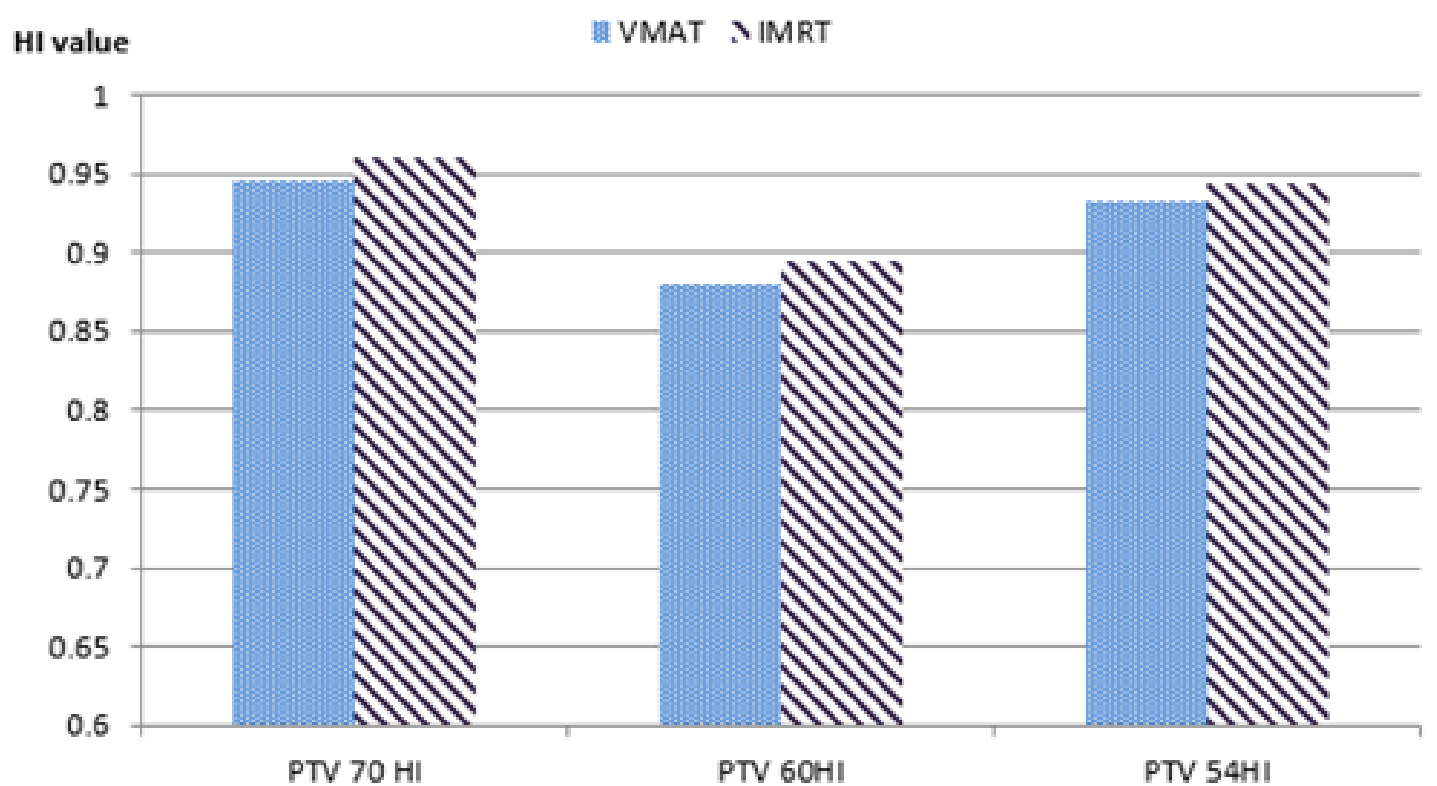

Figure 4: Comparison between VMAT and IMRT HI average for different target volumes.

Table 2: High target volume CN, GI and MU.

\begin{tabular}{|c|c|c|c|c|c|c|c|c|c|}
\hline & \multicolumn{3}{|c}{ CN } & \multicolumn{3}{c|}{ GI } & \multicolumn{2}{c|}{ MU } \\
\hline Case & VMAT & IMRT & VP & VMAT & IMRT & VP & VMAT & IMRT & VP \\
\hline 1 & 0.85 & 0.77 & -10.13 & 15.94 & 17.45 & 8.65 & 676 & 1972 & 65.72 \\
\hline 2 & 0.82 & 0.78 & -4.54 & 27.54 & 31.65 & 12.99 & 659 & 1936 & 65.96 \\
\hline 3 & 0.82 & 0.76 & -8.49 & 13.42 & 14.3 & 6.19 & 648 & 2005 & 67.68 \\
\hline 4 & 0.55 & 0.53 & -2.56 & 68.32 & 76.92 & 11.19 & 604 & 2412 & 74.96 \\
\hline 5 & 0.74 & 0.64 & -16.36 & 37.15 & 44.75 & 16.99 & 573 & 1973 & 70.96 \\
\hline 6 & 0.79 & 0.76 & -4.53 & 85.39 & 104.5 & 18.36 & 565 & 1987 & 71.57 \\
\hline 7 & 0.89 & 0.89 & -0.30 & 112.06 & 134.3 & 16.6 & 603 & 1758 & 65.70 \\
\hline 8 & 0.53 & 0.25 & -110.46 & 15.12 & 29.5 & 48.84 & 454 & 2436 & 81.36 \\
\hline 9 & 0.85 & 0.77 & -10.45 & 15.15 & 19.6 & 23.09 & 560 & 1748 & 67.96 \\
\hline 10 & 0.76 & 0.51 & -50.41 & 65.18 & 102.1 & 36.17 & 573 & 1994 & 71.26 \\
\hline 11 & 0.8 & 0.79 & -1.17 & 33.06 & 36.8 & 10.18 & 568 & 1660 & 65.78 \\
\hline AVG & 0.76 & 0.68 & 19.95 & 44.39 & 55.65 & 19.02 & 589 & 1989 & 69.90 \\
\hline
\end{tabular}

Table Abbreviations: CN: Conformity Number; GI: Gradient Index; MU: Monitor Units; VMAT: Volumetric Modulated Arc Therapy; IMRT: Intensity Modulated Radiation Therapy; VP: Variance Percentage; AVG: Average. 


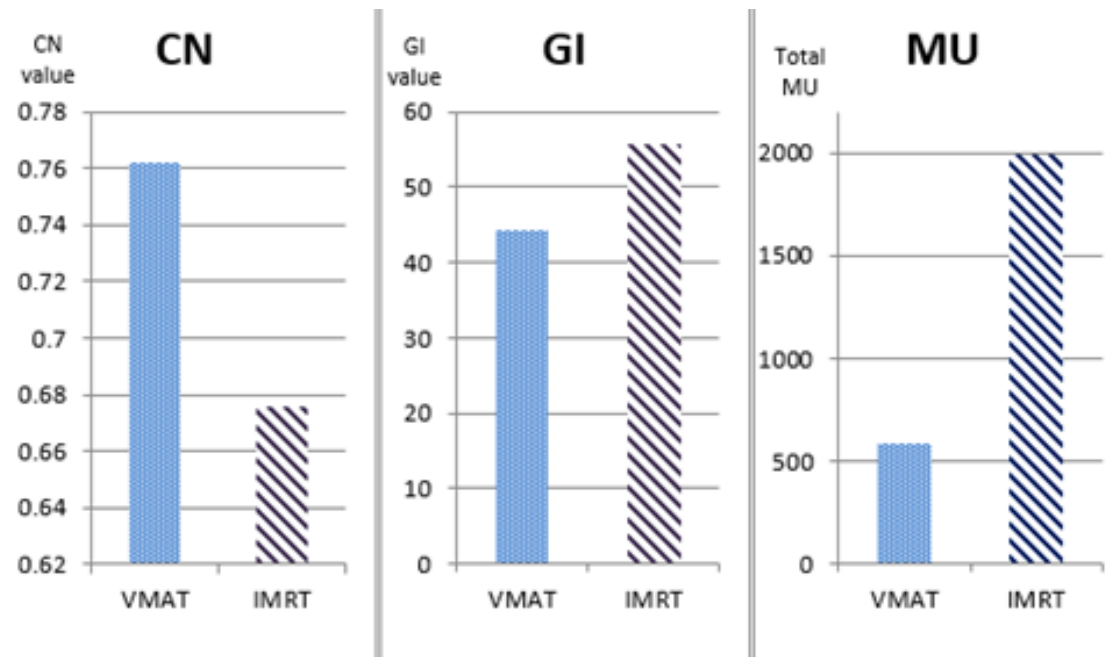

Figure 5: Comparison between VMAT and IMRT (CN and GI) average for high target volume and MU.
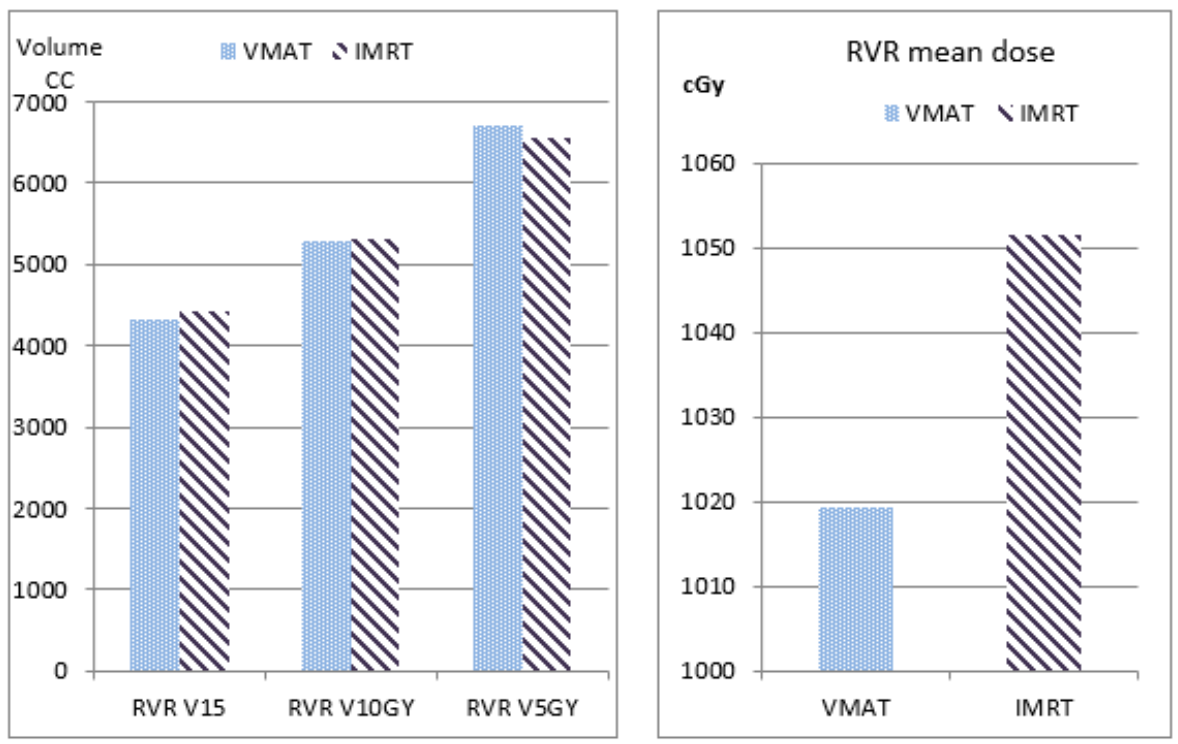

Figure 6: Comparison between VMAT and IMRT in RVR.

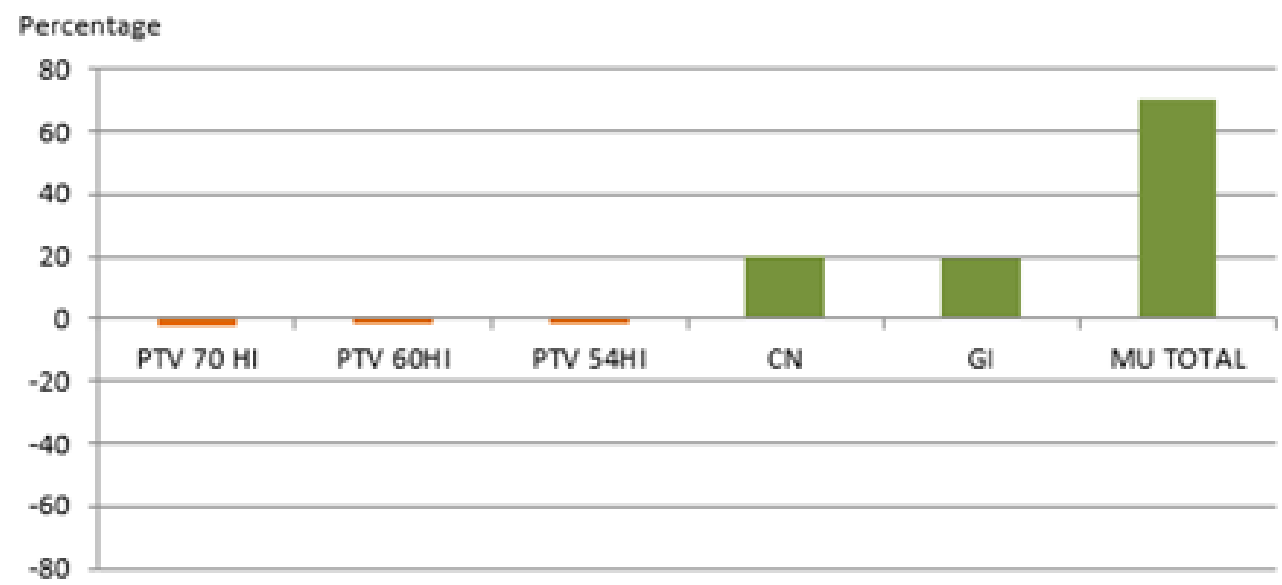

Figure 7: Chart of the variance percentage between the two techniques in HI, CI, CN, GI and MU. 


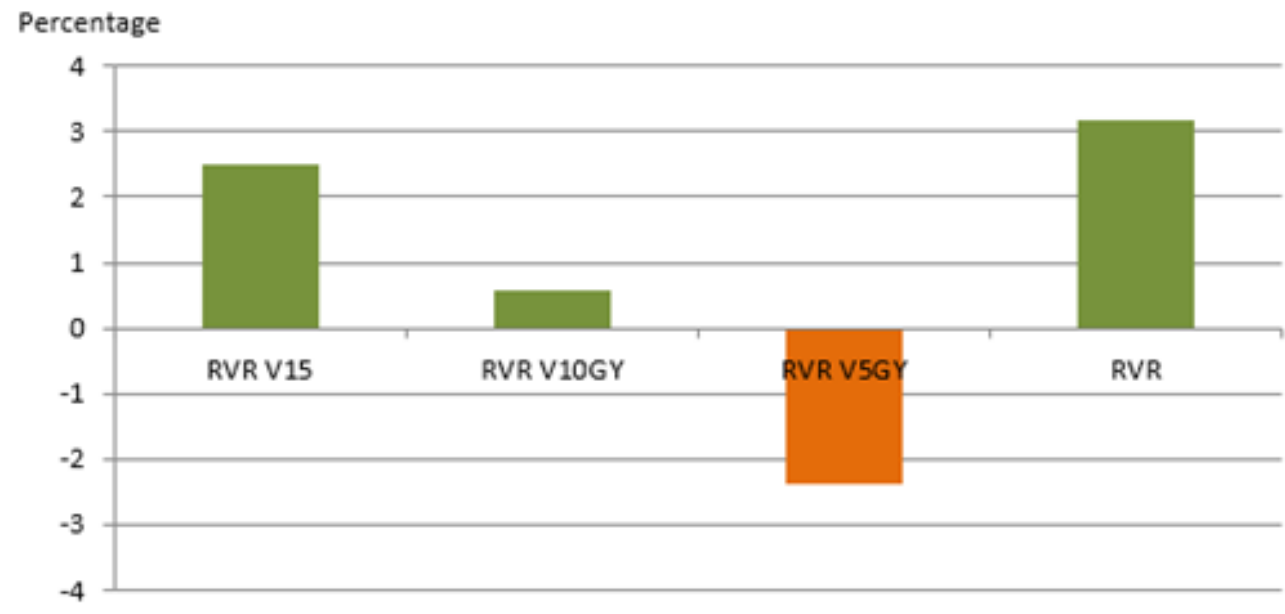

Figure 8: Chart of the variance percentage in RVR

Table 3: Low dose effect (RVR) comparison.

\begin{tabular}{|c|c|c|c|c|c|c|c|c|c|c|c|c|}
\hline & \multicolumn{3}{|c|}{ RVR V15 } & \multicolumn{3}{c|}{ RVR V10GY } & \multicolumn{3}{c|}{ RVR V5GY } & \multicolumn{3}{c|}{ RVR MEAN DOSE } \\
\hline Case & VMAT & IMRT & VP & VMAT & IMRT & VP & VMAT & IMRT & VP & VMAT & IMRT & VP \\
\hline 1 & 3482 & 3507 & 0.71 & 4281 & 4381 & 2.28 & 5496 & 5699 & 3.56 & 1066 & 1091 & 2.29 \\
\hline 2 & 4125 & 4287 & 3.78 & 5125 & 5179 & 1.04 & 6565 & 6399 & -2.59 & 869 & 923 & 5.85 \\
\hline 3 & 3635 & 3630 & -0.14 & 4460 & 4274 & -4.35 & 5502 & 5201 & -5.79 & 1041 & 1037 & -0.39 \\
\hline 4 & 3373 & 3385 & 0.35 & 3912 & 3964 & 1.31 & 4962 & 4879 & -1.70 & 1184 & 1161 & -1.98 \\
\hline 5 & 3710 & 3880 & 4.38 & 4738 & 4675 & -1.35 & 6093 & 6088 & -0.08 & 1204 & 1261 & 4.52 \\
\hline 6 & 5226 & 5475 & 4.55 & 6440 & 6675 & 3.52 & 8268 & 8074 & -2.4 & 895 & 947 & 5.49 \\
\hline 7 & 5877 & 5790 & -1.50 & 7332 & 7154 & -2.49 & 9554 & 8905 & -7.29 & 832 & 861 & 3.37 \\
\hline 8 & 4956 & 5087 & 2.58 & 5786 & 5926 & 2.36 & 7135 & 7072 & -0.89 & 1147 & 1153 & 0.52 \\
\hline 9 & 4686 & 4865 & 3.68 & 5751 & 5848 & 1.66 & 7348 & 6956 & -5.64 & 1085 & 1153 & 5.9 \\
\hline 10 & 5082 & 5197 & 2.21 & 6045 & 5984 & -1.02 & 7369 & 7087 & -3.98 & 920 & 940 & 2.13 \\
\hline 11 & 3455 & 3691 & 6.39 & 4227 & 4367 & 3.21 & 5442 & 5615 & 3.08 & 970 & 1041 & 6.82 \\
\hline AVG & 4327 & 4435 & $2.45 \%$ & 5281 & 5311 & $0.56 \%$ & 6703 & 6543 & $-2.16 \%$ & 1019 & 1051 & $3.14 \%$ \\
\hline
\end{tabular}

Table Abbreviations: RVR: Remaining Volume at Risk; VMAT: Volumetric Modulated Arc Therapy; IMRT: Intensity Modulated Radiation Therapy; VP: Variance Percentage; AVG: Average.

\section{Conclusion}

This study reported on two techniques that were comparable in homogeneity indices, while there is an advantage for VMAT in conformity number and gradient index over the IMRT. It is clear that VMAT is the first choice for easy delivery of treatment and timeless on the machine. In contrast, for the remaining volume at risk RVR, the study showed that VMAT has the upper hand in V15Gy, V10Gy and mean dose of RVR, while for the V5Gy, the IMRT has the least volume. Finally, the remaining volume at risk RVR evaluation is one of an advanced issues for plan quality that can estimate the risk of late effect, especially in the young patients.

\section{References}

1. Suditu M, Adam D Popa R, Ciocaltei V (2014) A vmat planning solution for neck cancer patients using the pinnacle3 planning system. Romanian Reports in Physics 66(2): 401-410.

2. Xie K, Chen J, Zou J, Chen L, Gong L (2017) Tumor volumes predict prognosis in head and neck cancer: A meta-analysis. Translational Cancer Research 6(4): 687-697.

3. Kumar SS, Vivekanandan N, Sriram P (2012) A study on conventional imrt and rapid arc treatment planning techniques for head and neck cancers. Reports of Practical Oncology \& Radiotherapy 17(3): 168-175.

4. Nicholls R (2003) A practical guide to intensity-modulated radiation therapy authors: Memorial sloan-kettering cancer center. Australasian Physics \& Engineering Sciences in Medicine 26(4): 200.

5. Ouyang Z, Liu Shen, Murray E, Kolar M, La Hurd D, et al. (2019) Evaluation of auto-planning in imrt and vmat for head and neck cancer. Journal of Applied Clinical Medical Physics 20(7): 39-47.

6. Akpochafor M, Adeneye S, Habeebu M, Abe G, Lawal R, et al. (2020) Evaluation of imrt and double-arc vmat treatment plans in head and neck cancer cases: Our experience at NSIA-LUTH cancer care. African Journal of Medical Sciences 3(1): 1-5.

7. Chen J, Dai J, Nobah A, Bai S, Bi N, et al. (2020) A special report on 2019 international planning competition and a comprehensive analysis of its results. Frontiers in Oncology 10: 571644. 
8. Mir R, Kelly SM, Xiao Y, Moore A, Clark CH, et al. (2020) Organ at risk delineation for radiation therapy clinical trials: Global harmonization group consensus guidelines. Radiotherapy and Oncology 150: 30-39.

9. Gozal F, Gondhowiardjo SA, Kodrat H, Wibowo WE (2020) Dosimetric analysis of three-dimensional conformal radiotherapy, intensitymodulated radiotherapy-step and shoot. Helical Tomotherapy, and volumetric modulated arc therapy in prostate cancer radiotherapy. Journal of Cancer Research and Therapeutics.

10. Chen SN, Ramachandran P, Deb P (2020) Dosimetric comparative study of 3DCRT, IMRT, VMAT, Ecomp, and Hybrid Techniques for Breast Radiation Therapy. Radiation Oncology Journal 38(4): 270-281.
11. Lee NY, Lu JJ (2012) Target volume delineation and field setup: A practical guide for conformal and intensity-modulated radiation therapy. Springer Science \& Business Media, pp. 1-11.

12. Al-Fishawy MM, Amin AES, Sallam AM, El-Sayed ESM (2018) Comparison of patient setup error effect on target evaluation parameters in 3D-CRT, IMRT and VMAT for Gioblastoma. Pan Arab Journal of Oncology, pp. 6471. 